\title{
Effects of Some Fluid Flow Possessions on Unsteady Conductive Free Convective Fluid on Account to Dissipative Porous Walls
}

\author{
Hussaini Abdullahi ${ }^{1}$ and Isah Bala Yabo ${ }^{2}$ \\ 1, Department of Mathematics, Sokoto State University, Sokoto, Nigeria \\ 2. Department of Mathematics, Usmanu Danfodiyo University, Sokoto, Nigeria
}

\begin{abstract}
The aspiration of this research paper is to study the influence of temperature dependent variable exponential viscosity, linear variable thermal conductivity and $\mathbf{n}^{\text {th }}$ order chemical reaction on MHD transitory natural convection heat and mass transfer through permeable medium. The ensuing nonlinear partial differential equations regulating the flow and the boundary conditions are concurrently reduced to nondimensional form by suitable transformations. The regulating non-dimensional equations are discretized using implicit finite difference technique and solved numerically. The outcomes of the numerical solutions are depicted graphically, during which skin friction, Nusselt and Sherwood numbers are set up in tabular form.
\end{abstract}

Keywords:Newtonian fluid, exponential variable viscosity, linear variable thermal conductivity, heat and mass transfer, MHD Flow, chemical reaction

\section{INTRODUCTION}

In the past decades speculative and cautious studies of viscous incompressibleNon-Newtonian fluids have been deeply investigated. Theoretical investigation of fluid flow past an infinite permeable channel is of very significant in lots of industrial contexts such as food processing, coating and polymer processing, paper production, hot rolling and glassfibre production. Nevertheless the study of heat and mass transfer flow of a viscous boundary layer over a stretching sheet in a porous medium has pertinent application in chemical engineering field and metallurgy as entreated by Batchelor and Shen [1], Anyakoha [2] and Andersson et al [3].Ramana and Chamkha [4] considered on the effects of first order chemical reaction andheat and mass transfer on steady two-dimensional natural convection flow with existence of MHD and viscous dissipation. They concluded that increasing the magnetic and porosity parameters decreases the velocity profiles while increasing the Eckert number increases both the velocity and temperature profiles. Animasaun [5] studied on steady state two-dimensional heat and mass transfer of viscous natural convective laminar incompressible fluid flow within a vertical porous medium with impressions of thermophoresis, temperature dependent variable viscosity and thermal conductivity, suction and $\mathrm{n}^{\text {th }}$ order chemical reaction. The analysis on consequence of Soret and Hall effects on MHD mixed convection past an infinite vertical porous plate was studied by Krishna et al. [6]. The unsteady MHD convective heat and mass transfer past a semi-infinite vertical porous plate with variable viscosity and thermal conductivity examined by Gnaneswara and Bhaska [7]. Oyem et al. [8] carried out a study on MHD free convective heat transfer on reacting flow over a vertical plate with constant thermal conductivity. The outcomes revealed that increasing the Eckert number in viscous dissipation increases the velocity and temperature profiles.

Magneto hydrodynamic (MHD) is a division of continuum mechanics which addresses the study of the flow of electrically conducting fluids in both electric and magnetic fields. Recently, there has been massive awareness in the study of MHD fluid flow and heat transfer in any medium due to its gigantic significant in many engineering analysis which includes MHD generators, plasma studies, nuclear reactors, geothermal energy extractions etc. Kisavaiah et al. [9] examined analytically on radiation absorption, chemical reaction and magnetic field effects on thefree Convection and mass transfer flow through porous medium with constant suction and constant heat flux. They exhibited that both velocity and temperature profiles increases with increasing chemical reaction parameter also increasing magnetic parameter causes a decrease in velocity profile. Durojaye at el. [10]worked on unsteady MHD free convective flow of an optically thick radiating viscous fluid over an infinite vertical plate in a consistent porous medium. Manjunatha and Gireesha [11]presented effects of variable viscosity and thermal conductivity on MHD flow and heat transfer of a dusty fluid. Ali et al. [12] applied finite element analysis to investigate the effect of variable viscosity on two-dimensional MHD incompressible Nanofluid flow by employing the Cattaneo-Christov model. Ali and Alam [13] critically analysed on the effects of Soret and Hall Effect on MHD flow over a vertical stretching sheet in a porous medium due to heat generation.

In industrial setup, high temperature is being expanded with lubricants, if they are exposed to uttermost conditions such as high temperature, pressure, high shear rate and external heating. According Anyakoha [2], Bachelor [14], Meyers [15] and host of Researchers, viscosity and thermal conductivity are the attributes which are most sensitive to temperature rise. The two-dimensional laminar free convective flow over a 
vertical plate with emphasis on effects of variable thermal conductivity and viscosity was investigated by Gbadeyan et al. [16]. Careful review of influence of temperature viscosity and thermal conductivity on heat transfer flow of unsteady micro polar fluid about a permeable cylinder with moving boundaries in the presence of magnetic field was carried out by Baruah and Hazarika [17]. Kiran at el. [18]emphasized on the effect of variable viscosity and thermal conductivity on MHD convective heat transfer of immiscible fluids in a vertical channel using Runge-Kutta $6^{\text {th }}$ order technique. Chandra Babu et al. [19] have performed the analysis of consequences of radiation and first order chemical reaction on unsteady two-dimensional viscous incompressible Nanofluid flow across a vertical cone embedded in porous medium. They deduced that the velocity profile increases with increasing variable viscosity parameter while temperature and concentration profiles decreases. Swain et al. [20] presented the MHD heat and mass transfer on a stretching sheet with variable fluid properties in a porous medium and concluded that variable thermal conductivity enhances the temperature profiles.

The analysis of chemically-reactive fluid flows is playing an important role, due to its practical purpose and relevant abundant applications in cooling tower dessign, geochemical transport in repositories and the dynamics of fog and mist composition, drying, distribution of temperature and moisture over agricultural fields and grooves of fruit trees, damage of crops due to freezing, evaporation at the interface of water body Patil [21]. Chemical reaction can be classified to be either homogeneous or heterogeneous process. Cussler [22]defined homogeneous reaction as one that occurs uniformly throughout a given phase whereas heterogeneous reaction is one that occurs in a restricted area or within the boundary of the phase. The order of the chemical reaction depends on many factors. The simplest chemical reaction is termed the first .order reaction where the variation of reaction is directly proportional to the species concentration. The simultaneous effect of buoyancy forces as a result of both thermal and mass diffusion in the presence of chemical reaction has significant relevant in the nuclear reactor safety and combustion system, metallurgical and chemical engineering. Uwanta and Halima [23] worked on the effects $\mathrm{n}^{\text {th }}$ order chemical reaction, radiation and variable thermal conductivity of natural convection unsteady flow of an incompressible viscous fluid. They established that increasing thermal Grashof number, solutal Grash of number and thermal conductivity parameter increases velocity profiles while increasing magnetic parameter, chemical reaction, Prandtl number, and Schmidt number decreases velocity profiles. Raghunath et al. [24] carefully talked about the effect of heat and mass transfer on MHD flow of an incompressible fluid having viscous and plastic properties past an infinite oscillating vertical porous plate with uniform magnetic field. They found out that, the velocity profile decreases with increase of Prandtl number and porosity parameter and decreases with increasing magnetic parameter. Quite recently,
Mjankwi et al. [25] comprehensively investigated on unsteady MHD flow of Nanofluid with variable attributes across an inclined stretching sheet in the presence of thermal radiation and chemical reaction and the analysis revealed that increase in the variable thermal conductivity and variable viscosity parameters increases temperature and concentration profiles. Uwanta and Murtala [26] have executed the study of the effects of thermal diffusion, variable viscosity and thermal conductivity on unsteady hydrmagnetic flow of reactive viscous fluid in a vertical channel. Uwanta and Sani [27] examined on effects of variable thermal conductivity and heat source of unsteady laminar free convection flow of dissipative fluid over an infinite vertical isothermal porous plate in the presence of uniform magnetic field. Jha et al. [28] critically examined free convective unsteady Couette flow. The study revealed that magnetic parameter produces Lorentz force that oppose the flow of an electrically carrying fluid.

In all the aforementioned analysis, the thermo physical properties notably fluid viscosity was assumed as reciprocal function of temperature. The focus of this manuscript is to study the set-up of temperature dependent variable viscosity and thermal conductivity on MHD unsteady two-dimensional streamlined flow of incompressible fluid past an infinite channel. In this investigation the viscosity and thermal conductivity are presumed as the exponential and a reciprocal function of temperature respectively.

\section{II.MATHEMATICAL FORMULATIONS}

Consider the unsteady viscous temperature dependent heat mass transfer with nth order chemical reaction over a vertical channel formed by two finite parallel plates separated by a distance $H$. The effects of uniform magnetic field, permeability, suction/injection, heat source, radiation and Soret effects are considered for the study. The geometry of the problem is shown in Fig. 1. In this analysis, $x^{\prime}$ and $y^{\prime}$ a -axis are taken such that the flow is assume to be in $x$-direction vertically upward along the channel walls of plate and $y^{\prime}$ axis normal to the plate. The fluid obeys the Boussinesq approximation. Under this assumption the governing boundary layer equations of continuity, momentum, energy and concentration equations in dimensional form are:
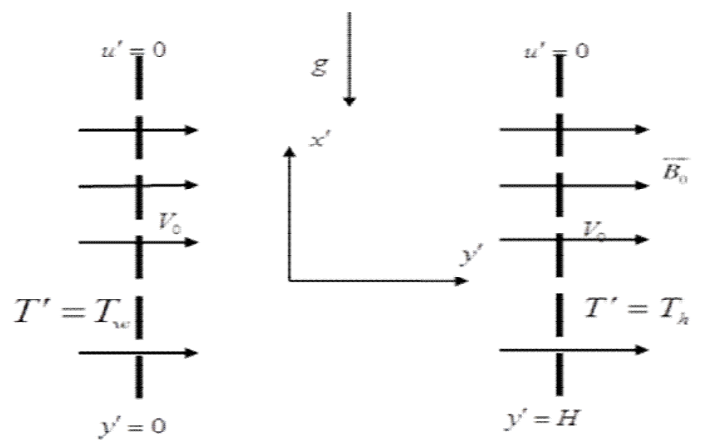

Figure 1: Geometry of the Problem 


$$
\begin{aligned}
& \frac{\partial v^{\prime}}{\partial y^{\prime}}=0 \\
& \frac{\partial u^{\prime}}{\partial t^{\prime}}+v^{\prime} \frac{\partial u^{\prime}}{\partial y^{\prime}}=\frac{\mu_{0}}{\rho} \frac{\partial}{\partial y}\left\{e^{-\lambda\left(T^{\prime}-T_{h}^{\prime}\right)} \frac{\partial u^{\prime}}{\partial y^{\prime}}\right\}+g \beta\left(T^{\prime}-T_{h}^{\prime}\right)+g \beta^{*}\left(C^{\prime}-C_{h}^{\prime}\right) \\
& -\frac{\sigma B_{0}^{2}}{\rho} u^{\prime}-\frac{v}{k^{\prime}} u^{\prime} \\
& \frac{\partial T}{\partial t^{\prime}}+v^{\prime} \frac{\partial T}{\partial y^{\prime}}=\frac{k_{0}}{\rho c_{p}} \frac{\partial}{\partial y^{\prime}}\left\{\left[1+m\left(T-T_{h}^{\prime}\right)\right] \frac{\partial T}{\partial y^{\prime}}\right\}-\frac{1}{\rho c_{p}} \frac{\partial q_{r}}{\partial y^{\prime}}+\frac{\mu}{c_{p}}\left(\frac{\partial u^{\prime}}{\partial y^{\prime}}\right)^{2} \\
& +\frac{Q_{0}}{\rho c_{p}}\left(T^{\prime}-T_{h}^{\prime}\right) \\
& \frac{\partial C^{\prime}}{\partial t^{\prime}}+v^{\prime} \frac{\partial C^{\prime}}{\partial y^{\prime}}=D \frac{\partial^{2} C^{\prime}}{\partial y^{\prime 2}}-R\left(C^{\prime}-C_{h}^{\prime}\right)^{n}+\sigma_{1} \frac{\partial^{2} T^{\prime}}{\partial y^{\prime 2}}
\end{aligned}
$$

The accompanying initial and boundary conditions are prescribed as follows:

$$
\begin{aligned}
& t^{\prime} \leq 0: u^{\prime}=0, T^{\prime}=T_{h}, C^{\prime}=C_{h} \text { for } 0 \leq y^{\prime} \leq H \\
& t^{\prime}>0:\left\{\begin{array}{l}
u^{\prime}=0, T^{\prime}=T_{\omega}, C^{\prime}=C_{\omega} \text { at } y^{\prime}=0 \\
u^{\prime}=0, T^{\prime}=T_{h}, C^{\prime}=C_{h} \text { at } y^{\prime}=H
\end{array}\right.
\end{aligned}
$$

Adopting Brewster [29], the radiative heat flux $q_{r}^{\prime}$ is given by

$q_{r}^{\prime}=-\frac{4 \sigma^{*} \partial T^{4}}{3 k^{*} \partial y^{\prime}}$

where $\sigma^{*}$ is the Stefan-Boltzmann constant and $k^{*}$ is the mean absorption coefficient. Assuming that the difference in temperature within the flow is such that $T^{4}$ can be expressed as a linear combination of the temperature, we expand $T^{4}$ in Taylor's series about $T_{h}$ as follows

$$
T^{4}=T_{h}^{4}+4 T_{h}^{3}\left(T-T_{h}\right)+6 T_{h}^{2}\left(T-T_{h}\right)^{2}+\ldots
$$

And neglecting higher order terms beyond the first degree $\left(T-T_{h}\right)$ in, we obtain

$T^{4}=-3 T_{h}^{4}+4 T_{h}^{3} T$

Differentiating equation (2.6) with respect to $y^{\prime}$ we obtain

$$
\frac{\partial q_{r}^{\prime}}{\partial y^{\prime}}=-\frac{16 T_{h}^{3} \sigma^{*} \partial^{2} T}{3 k^{*} \partial y^{2}}
$$

Invoking equations (2.6), (2.8) and (2.9) in Equation (2.3) we get

$$
\begin{aligned}
& \frac{\partial T}{\partial t^{\prime}}+v^{\prime} \frac{\partial T}{\partial y^{\prime}}=\frac{k_{0}}{\rho_{p}} \frac{\partial}{\partial y^{\prime}}\left\{\left[1+m\left(T^{\prime}-T_{h}^{\prime}\right)\right] \frac{\partial T^{\prime}}{\partial y^{\prime}}\right\}+\frac{1}{\rho c_{p}} \frac{16 T_{h}^{3} \sigma^{*}}{3 k^{*}} \frac{\partial^{2} T}{\partial y^{2}}+\frac{\mu}{c_{p}}\left(\frac{\partial u}{\partial y^{\prime}}\right)^{2} \\
& +\frac{Q_{0}}{\rho c_{p}}\left(T^{\prime}-T_{h}^{\prime}\right)
\end{aligned}
$$

To exhibit solutions in non-dimensional form, we introduced the following non-dimensional variables

$$
\begin{aligned}
& x^{\prime}=\frac{x}{H}, y^{\prime}=\frac{y G^{1 / 4}}{H}, t^{\prime}=\frac{t H^{2} G^{-1 / 2}}{v}, u^{\prime}=\frac{u G^{-1 / 2}}{v}, G=\frac{g \beta H^{2}\left(T_{\omega}^{\prime}-T_{h}\right)}{v^{2}}, \phi=\frac{Q_{f} H^{2}}{\rho_{p} \nu G^{1 / 2}}, \\
& T=\frac{T^{\prime}-T_{h}^{\prime}}{T_{\omega}^{\prime}-T_{h}}, C=\frac{C^{\prime}-C_{h}^{\prime}}{C_{\omega}^{\prime}-C_{h}^{h}}, \mathrm{Pr}=\frac{v}{\alpha}, F=\frac{k k}{4 \sigma_{s} T_{h}^{3}}, S c=\frac{v}{D}, F c=\frac{u_{0}^{2}}{c_{p}\left(T_{\omega}^{\prime}-T_{h}^{\prime}\right)}, N=\frac{\beta\left(C_{\omega}^{\prime}-C_{h}^{\prime \prime}\right)}{\beta\left(T_{\omega}^{\prime}-T_{h}^{\prime}\right)} \\
& M=\frac{\sigma B_{0}^{2}}{u_{0}^{2}}
\end{aligned}
$$

By bringing in the above non-dimensional equation (2.11) in equations $(2.1),(2.2)$ and $(2.10)$, they are brought down to the non-dimensional form as:

$$
\begin{aligned}
& \frac{\partial u}{\partial t}+\eta \frac{\partial u}{\partial y}=\exp (-\lambda I)\left[\frac{\partial^{2} u}{\partial y^{2}}-\lambda \frac{\partial u}{\partial y} \frac{\partial T}{\partial y}\right]+T+N C-\left(M+\frac{1}{D a \operatorname{Re}}\right) u \\
& \operatorname{Pr}\left[\frac{\partial T}{\partial t}+\eta \frac{\partial T}{\partial y}\right]=\left[1+\gamma T+\frac{4}{3 F}\right] \frac{\partial^{2} T}{\partial y^{2}}+\gamma\left(\frac{\partial T}{\partial y}\right)^{2}+\operatorname{Pr} E c\left(\frac{\partial u}{\partial y}\right)^{2}+\operatorname{Pr} \phi T
\end{aligned}
$$

$$
\begin{aligned}
& S c\left[\frac{\partial C}{\partial t}+\eta \frac{\partial C}{\partial y}\right]=\frac{\partial^{2} C}{\partial y^{2}}-S c K r C^{n}+S c S r \frac{\partial^{2} T}{\partial y^{2}}
\end{aligned}
$$

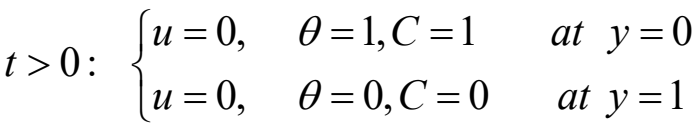

\section{METHOD OF SOLUTION}

The governing partial differential Equations (2.12)-(2.14) of momentum, energy and concentration together with the boundary conditions (2.15) are solved numerically by using implicit finite difference scheme with the help of Matlab software. We used backward difference for time derivatives and first and second order for space derivatives are approximated by the central difference formula to obtained finite difference equations which are transformed into a system of linear algebraic equations in the tri-diagonal form corresponding to (2.12)-(2.14) as follows:

$$
-r_{1} r_{5} u_{i-1}^{j+1}+\left(1+2 r_{1} r_{5}\right) u_{i}^{j+1}-r_{1} r_{5} u_{i-1}^{j+1}=r_{2} r_{5} u_{i-1}^{j}+\left(1+\eta r_{4}-2 r_{2} r_{5}-\Delta t M-\frac{\Delta t}{D a \mathrm{Re}}\right) u_{i}^{j}
$$


$+\left(r_{2} r_{5}-\eta r_{4}\right) u_{i+1}^{j}+\Delta t T_{i}^{j}+\Delta t N C_{i}^{j}$

$-r_{1} r_{6} T_{i-1}^{j+1}+\left(\operatorname{Pr}+2 r_{1} r_{6}\right) T_{i}^{j+1}-r_{1} r_{6} T_{i+1}^{j+1}=r_{2} r_{6} T_{i-1}^{j}+\left(\operatorname{Pr}+2 r_{2} r_{6}+\eta r_{4} \operatorname{Pr}+\Delta t \operatorname{Pr} \phi\right) T_{i}^{j}$

$+\left(r_{2} r_{6}-\eta r_{4} \operatorname{Pr}\right) T_{i+1}^{j}+\gamma r_{4}\left(T_{i+1}^{j}-T_{i-1}^{j}\right)^{2}+r_{4} \operatorname{Pr} E c\left(u_{i+1}^{j}-u_{i-1}^{j}\right)^{2}+\Delta t \operatorname{Pr} b\left(u_{i}^{j}\right)^{2}$

$-r_{1} C_{i-1}^{j+1}+\left(S c+2 r_{1}\right) C_{i}^{j+1}-r_{1} C_{i+1}^{j+1}=-r_{2} C_{i-1}^{j}+\left(S c+\eta S c r_{4}-2 r_{2}\right) C_{i}^{j}$

$+\left(r_{2}-\eta S c r_{4}\right) C_{i+1}^{j}-\Delta t S c K r\left(C_{i}^{j}\right)^{n}$

where

$r 1=\frac{\theta \Delta t}{\Delta y^{2}} ; r 2=\frac{(1-\theta) \Delta t}{\Delta y^{2}} ; r 3=\frac{\Delta t}{\Delta y} ; r 4=\frac{\lambda \Delta t}{4(\Delta y)^{2}} ; r 5=1+\gamma T+\frac{4}{3 F} ; r 6=\frac{\gamma \Delta t}{(\Delta y)^{2}}$

\section{RESULTS AND DISCUSSION}

The numerical computations of dimensionless velocity, temperature and concentration profiles have been carried out for different values of the parameters. The velocity, temperature and concentration profiles obtained in dimensionless form are presented in Figures (2-13). The Prandtl number for the working fluids are taken to be $\operatorname{Pr}=0.71$ and $\operatorname{Pr}=7.0$ which corresponds to air and water respectively. The values of Schmidt number $(\mathrm{Sc})$ were chosen to be $\mathrm{Sc}=0.22,0.78,0.94$, consituting diffusing chemical species of most usual concern in air like $\mathrm{H}_{2}, \mathrm{NH}_{3} \mathrm{CO}_{2}$ respectively whiles other parameters are chosen discretionary. The effect of buoyancy ratio parameter(N) is displayed in Figures 2(a) and 2(b). It is noticed that the velocity increases with increasing values of the buoyancy ratio parameter. It is also observed that velocity is higher in air $(\mathrm{Pr}=0.71)$ compare to that of water $(\operatorname{Pr}=7.0)$. Figures3(a) and 3(b) depict the distribution of transient velocity against $y$ for various $\mathrm{Schmidt}(\mathrm{Sc})$ values. The Schmidt number is the ratio of the momentum diffusivity to the mass diffusivity. Physically, it relates the relative thickness of the hydrodynamic boundary layer and mass- transfer boundary layer. It is seen that as Schmidt (Sc) number increases the velocity field decreases. The effects of the radiation parameter $(\mathrm{F})$, on the transient velocity profiles are display on Figures 4 (a) and 4 (b). It can be inferred from the Figures that an increase in thermal radiation parameter $(\mathrm{F})$ produces significant increase in the thermal condition of the fluid and its thermal boundary layer. Through the buoyancy effect the increase in fluid temperature induces more flow in the boundary layer causing the velocity to increase. In addition the hydrodynamic boundary layer thickness increases as a result increasing the radiation parameter $(F)$. Substituting different values of the viscosity parameter $(\lambda=5,10,15,20)$ we observe that the velocity profile increases with decreasing of $\lambda$ as depicted in Figure 5(a) and 5(b). It is also noticed that velocity is higher in injection compared to velocity in suction. Figures 6(a) and 6(b) represent the effect of magnetic field parameter; they show that the momentum is decreasing due to the Lorentzforces which have the capacity to act against the flow. The Figures further revealed that velocity is higher in injection compared to suctionin both fluid parameters.
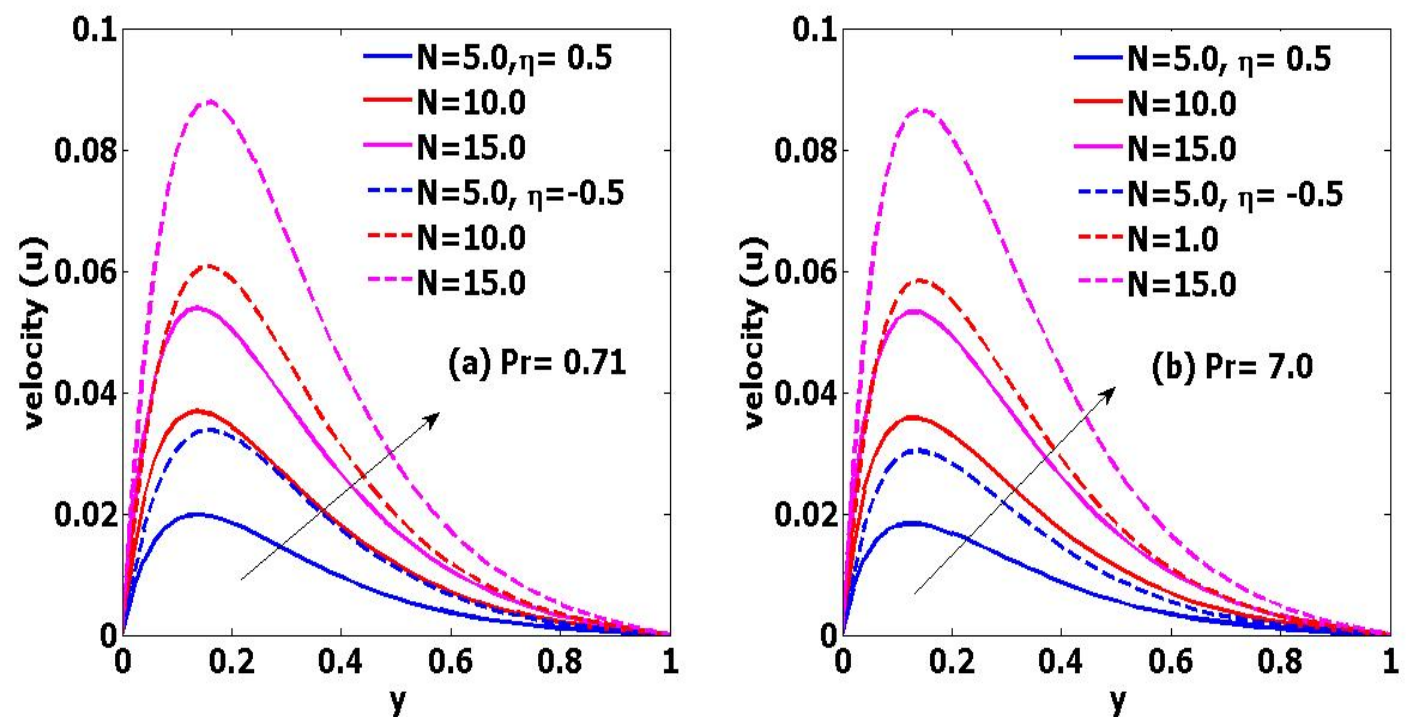

Figure2: Effect of Buoyancy parameter $(\mathrm{N})$ on velocity profiles 

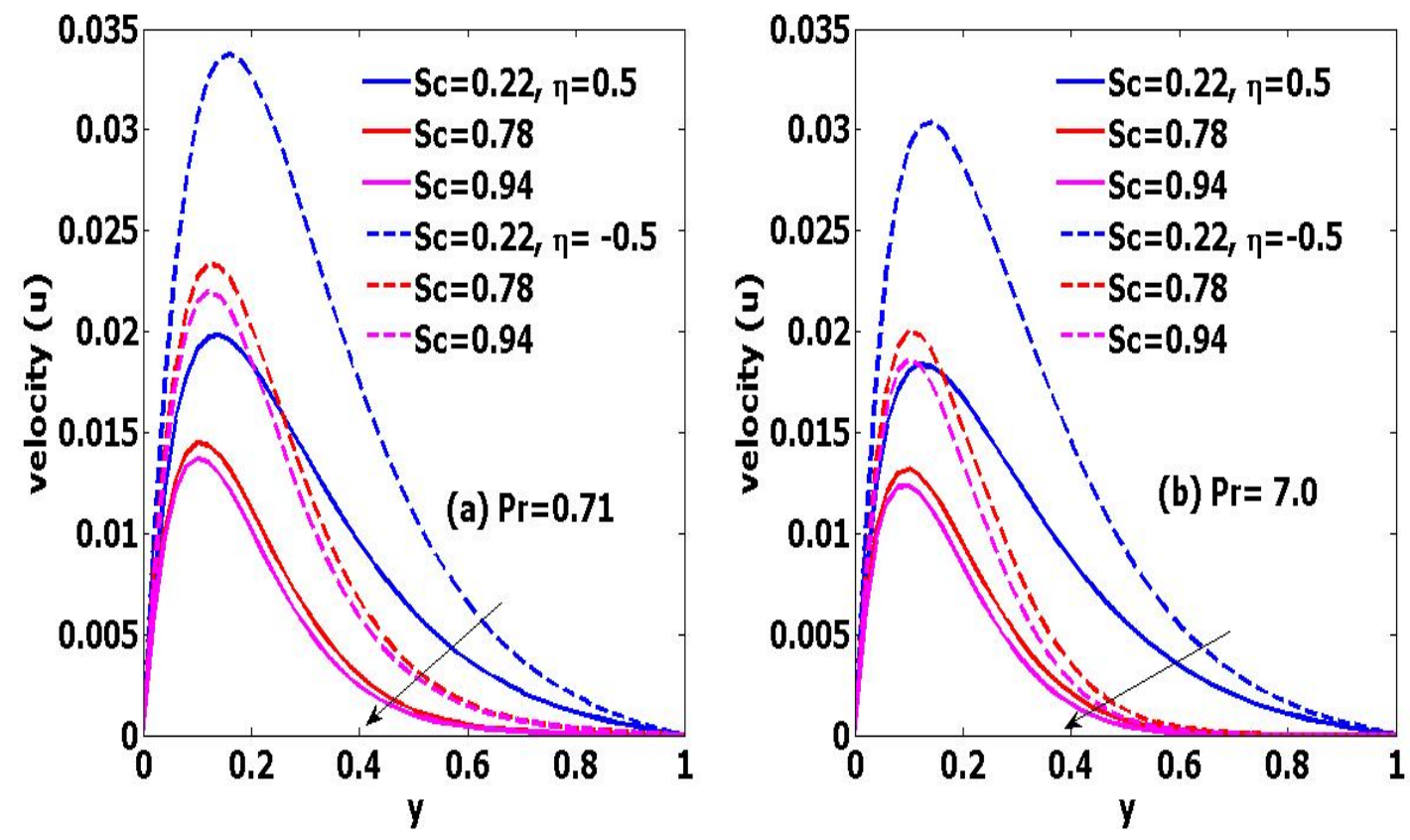

Figure 3: Effect of Schmidt number (Sc) on velocity profiles
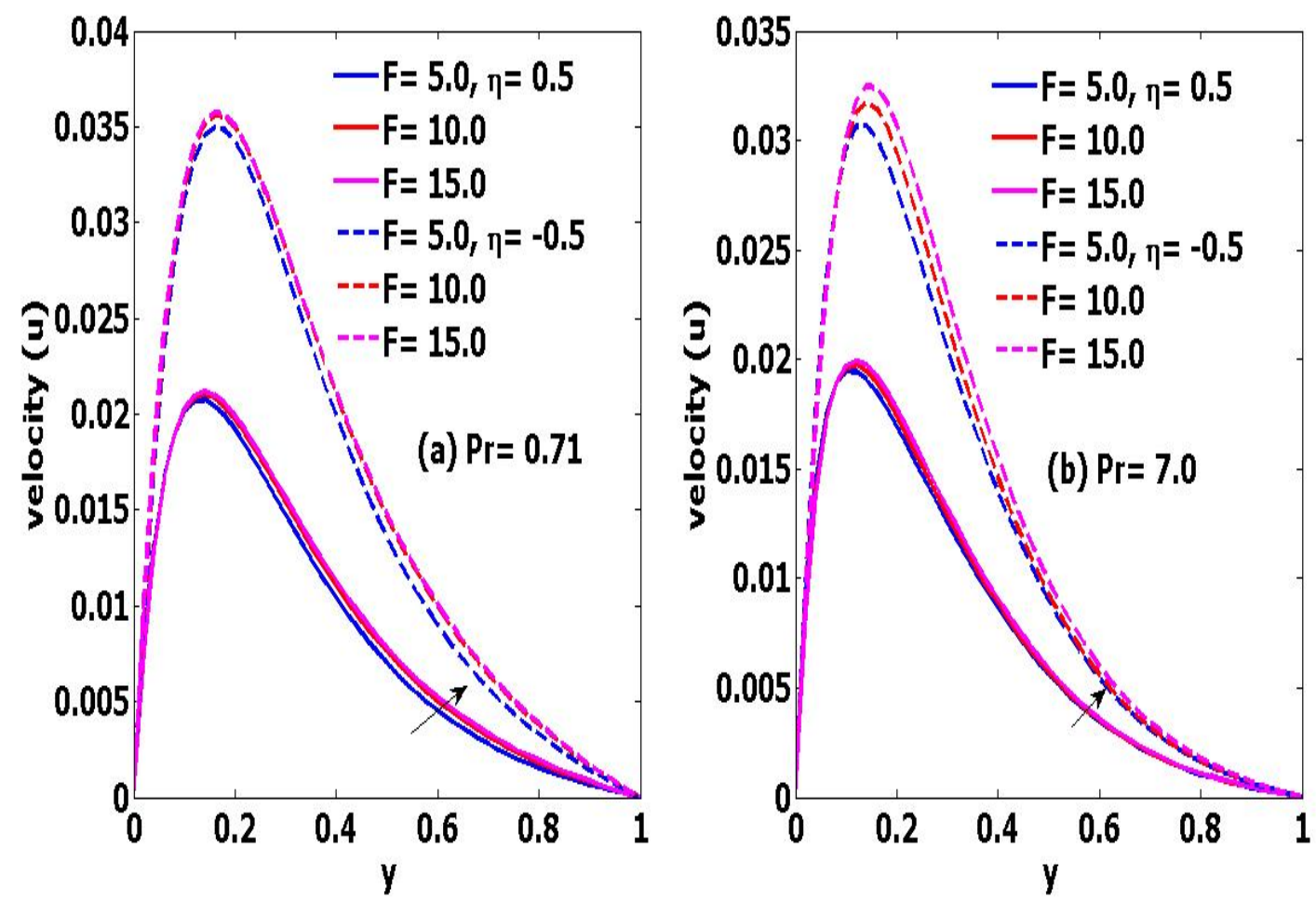

Figure 4: Effect of Radiation parameter (F) on velocity profiles 

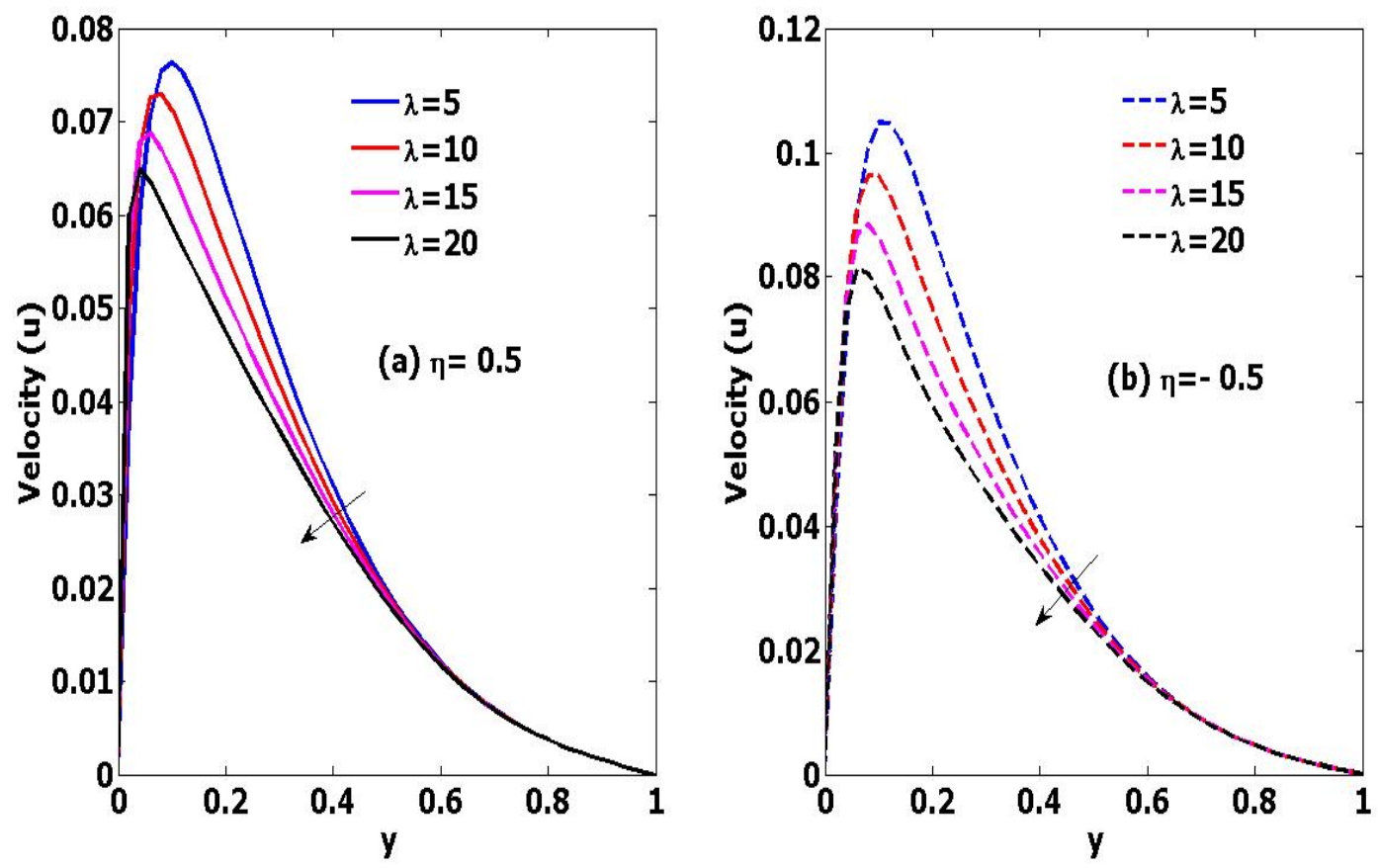

Figure 5: Effect of variable Viscosity parameter $(\lambda)$ on velocity profiles
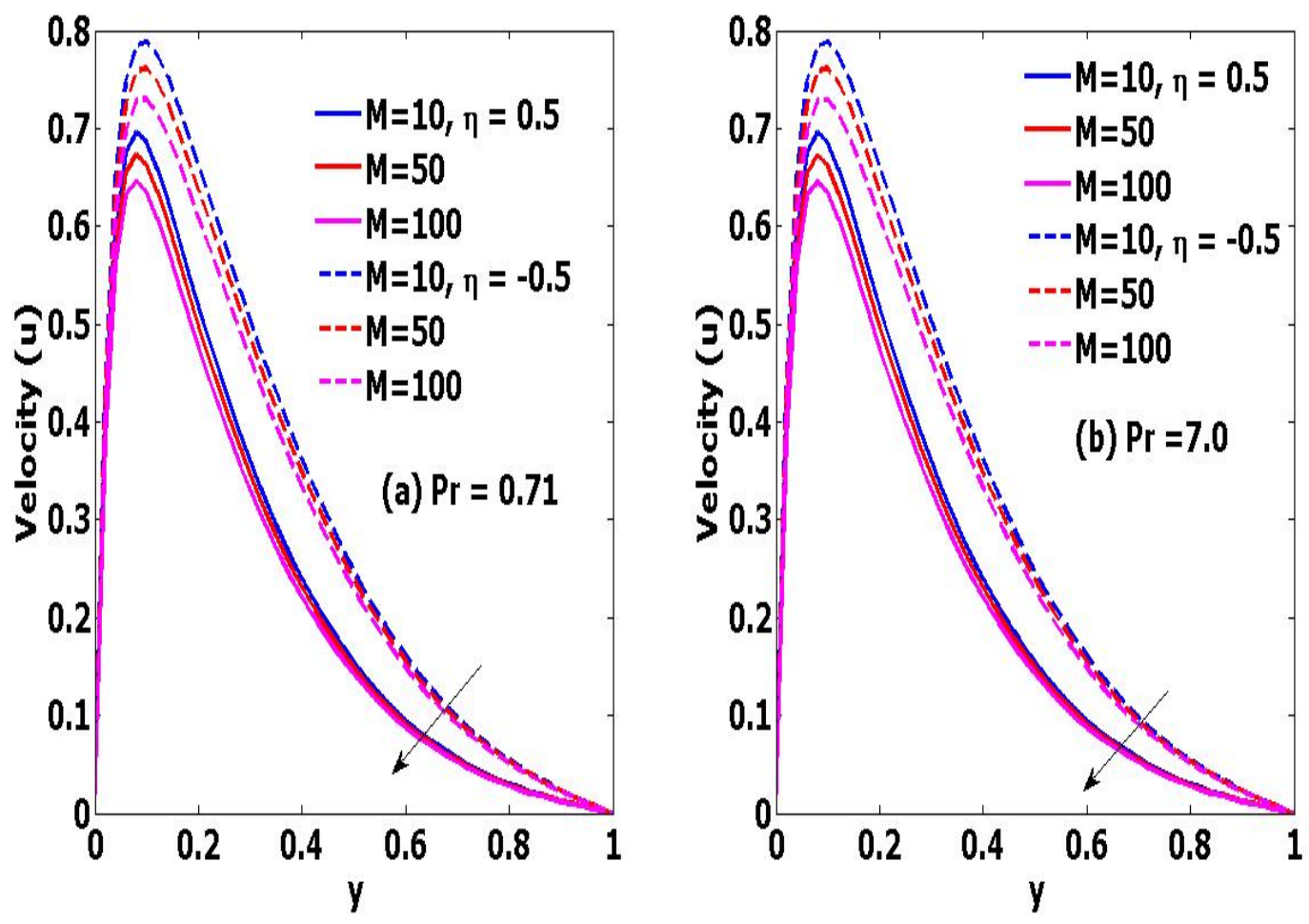

Figure 6: Effect of Magnetic parameter (M) on velocity profiles

The effects of various parameters on temperature profiles in the boundary layer are illustrated in Figures 7(a) and (b) through Figures 11(a) and (b). For different values of radiation parameter $(\mathrm{F})$, the temperature profiles are plotted in
Figures 7(a) and (b). Here we observed that as the value of $F$ increases the temperature elevates with an increasing in the thermal boundary layer thickness. However, is also observed from Figure 7(b) there is decay in the values of temperature 
along the space coordinate $0.64 \leq y \leq 1$. Typical variations of temperature profile along the coordinate y are depicted in Figures 8(a) and (b) for different values of Prandtl number (Pr). The result posits that an increase of Pr results in a decreasing the thermal boundary layer thickness and more uniform temperature distribution across the boundary layer. This is true since smaller values of $\operatorname{Pr}$ are equivalent to increasing the thermal conductivities and hence, heat is able to diffuse away from the heated surface faster for higher values of Pr. Figures 9(a) and (b) exhibit the effect of variable thermal conductivity parameter $(\gamma)$ on temperature profiles in the boundary layer. An increase in the value of $\gamma$ accelerates the magnitude of temperature distribution. It is further revealed from the Figures that $\gamma$ is more pronounced in air $(\operatorname{Pr}=0.71)$ compared to that of water $(\operatorname{Pr}=7.1)$. Variation of temperature profiles for different values of heat generation parameter $(\phi)$ is portraying in Figure 10(a) and (b) for suction and injection. It is observed from the Figures that velocity enhances on increasing heat generation parameter $(\phi)$.
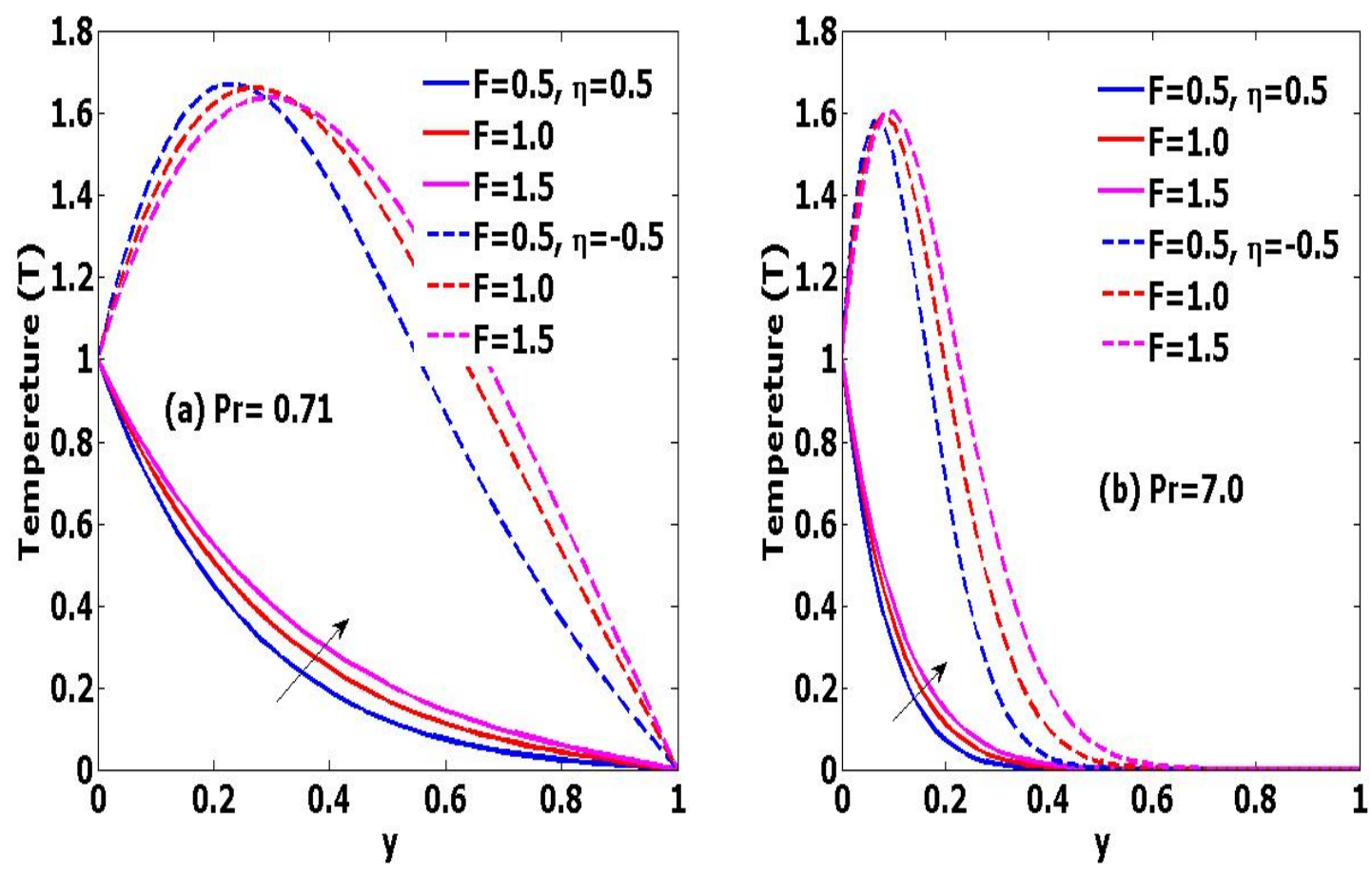

Figure 7: Effect of Radiation parameter ( $F$ ) on temperature profiles
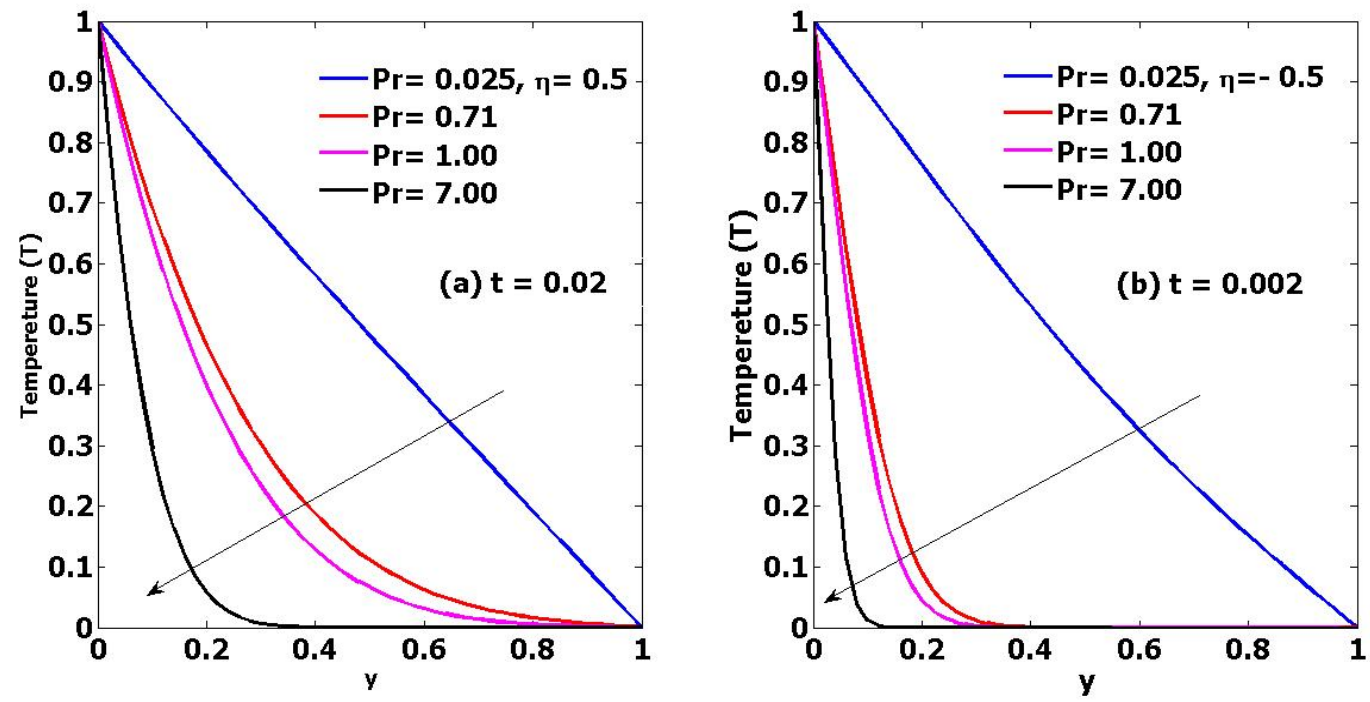

Figure 8: Effect of Prandtl number ( $\mathrm{Pr}$ ) on temperature profiles 

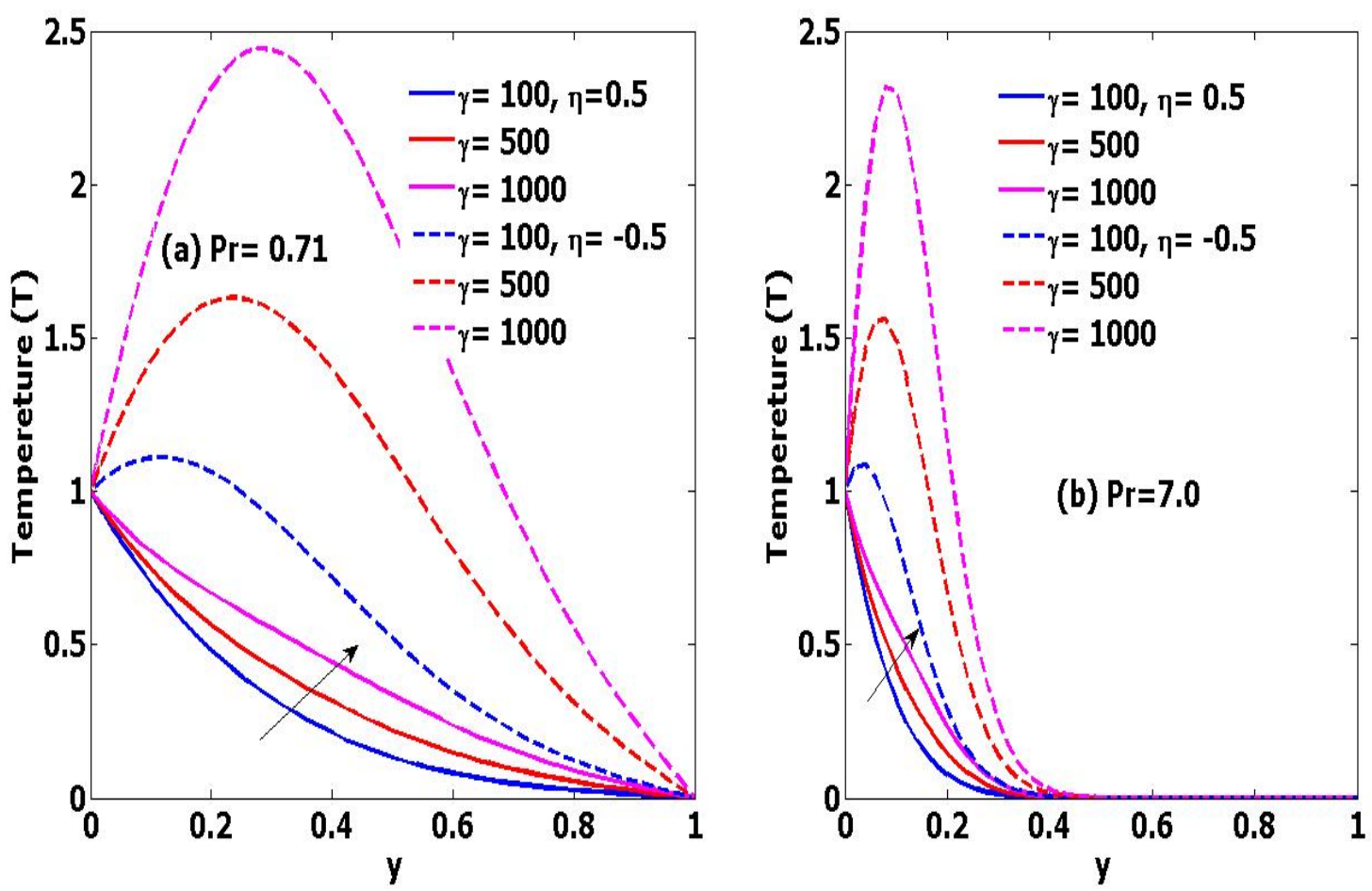

Figure 9: Effect of Thermal conductivity $(\gamma)$ on temperature profiles
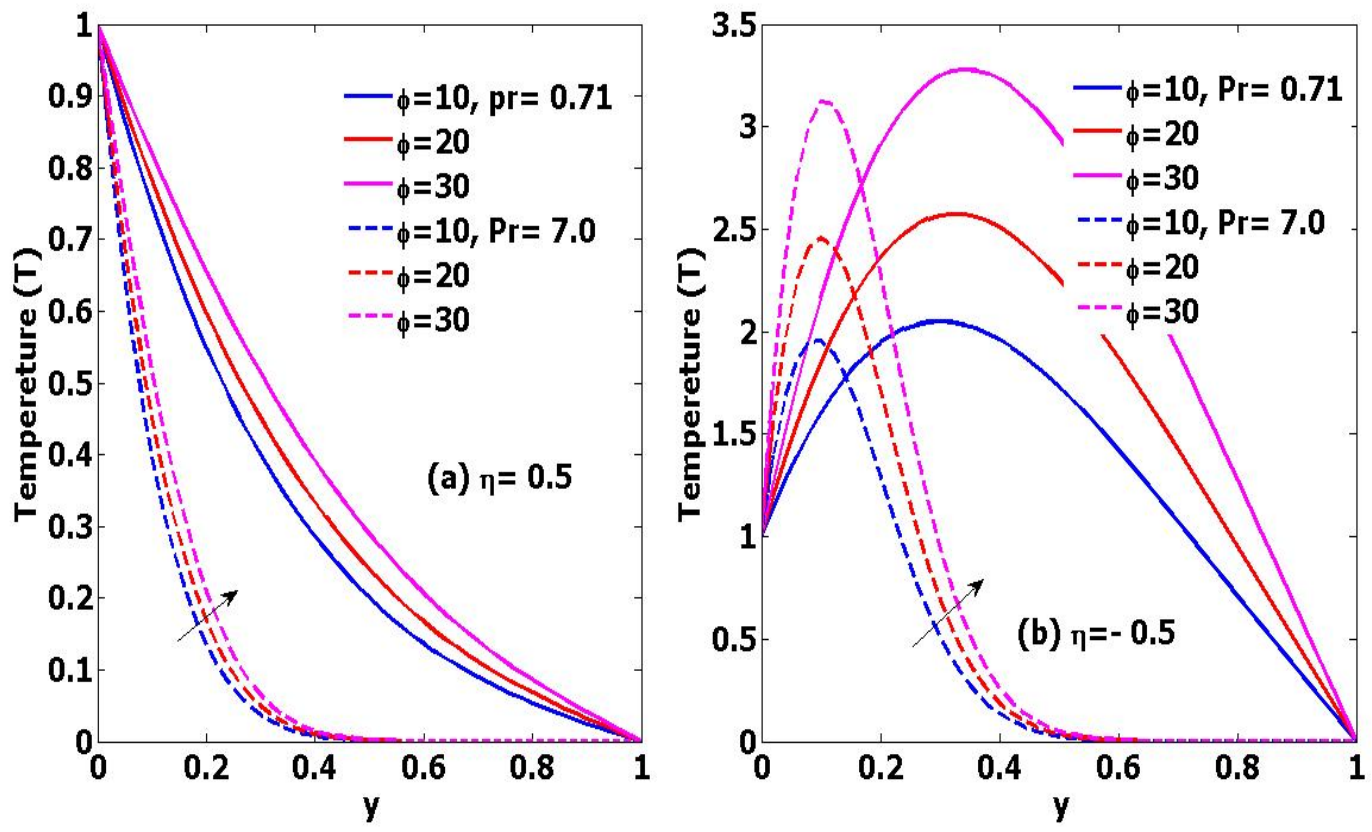

Figure 10: Effect of Heat source parameter $(\phi)$ on temperature profiles

Figures 11(a) and (b) highlight the concentration profiles across the boundary layer for various values of Schmidt number $(\mathrm{Sc})$. The Figures show that an increasing in Sc results in retarding the concentration distribution because the smaller values of $\mathrm{Sc}$ are equivalent to elevating the chemical molecular diffusivity. The variation of chemical reaction parameter $(\mathrm{Kr})$ on the concentration profiles is shown in Figures 12(a) and (b). It is noticed that increase in 
the chemical reaction parameter $(\mathrm{Kr})$ contributes in the decreases in the concentration of the fluid media. The effect of order of chemical reaction (n) on the concentration profiles is shown in Figures 13(a) and (b). While all other involving parameters in the concentration field are held constant, increase in concentration field is observed.
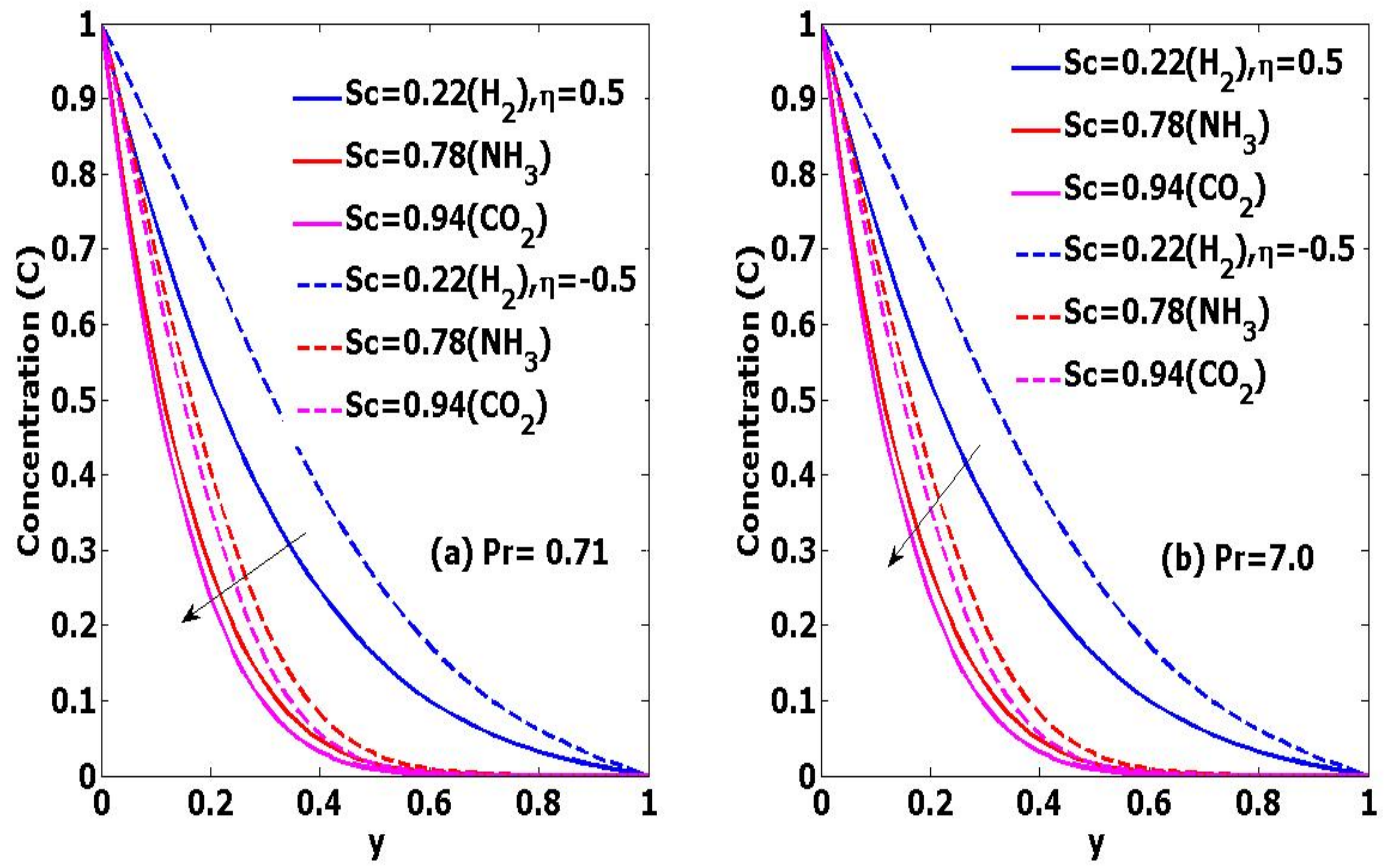

Figure 11: Effect of Schmidt number ( $S c$ ) on Concentration profiles
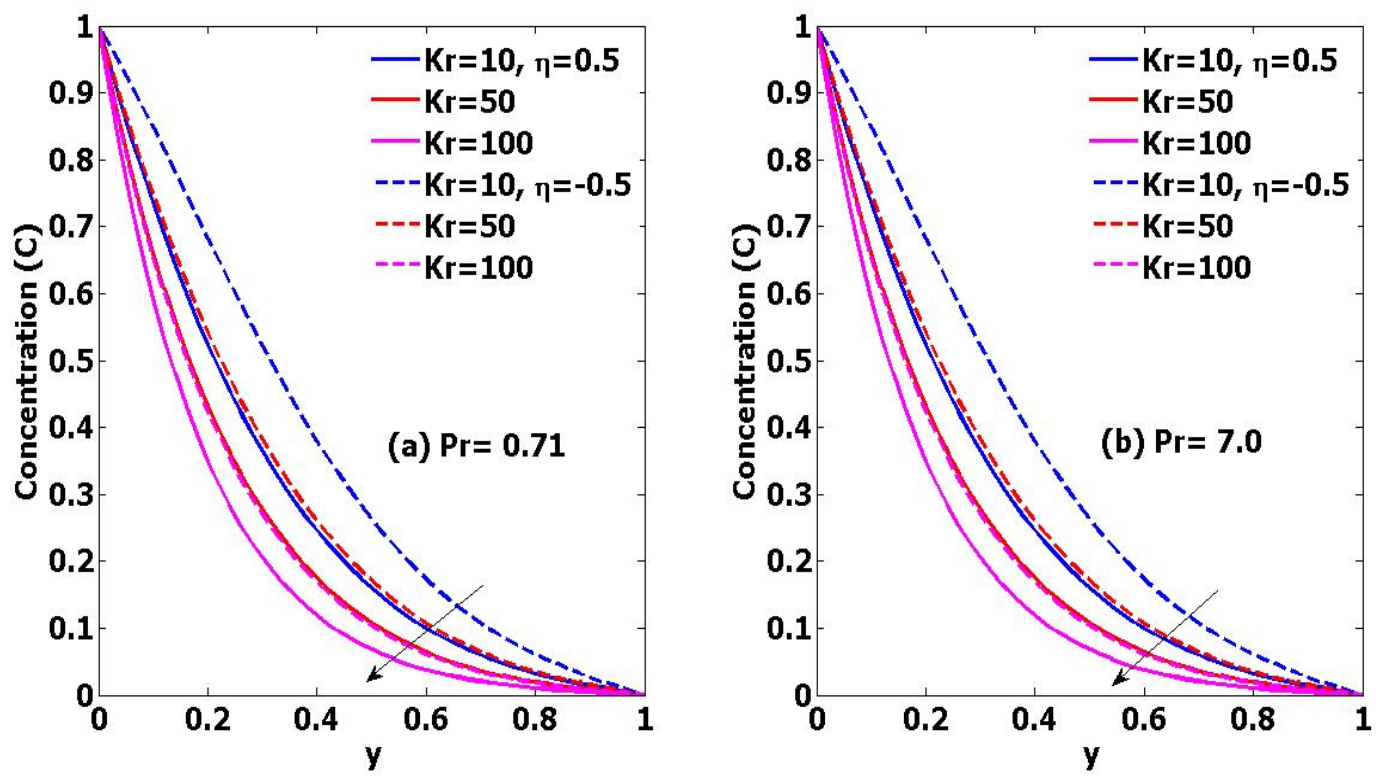

Figure 12: Effect of Chemical reaction parameter $(K r)$ on Concentration profiles 

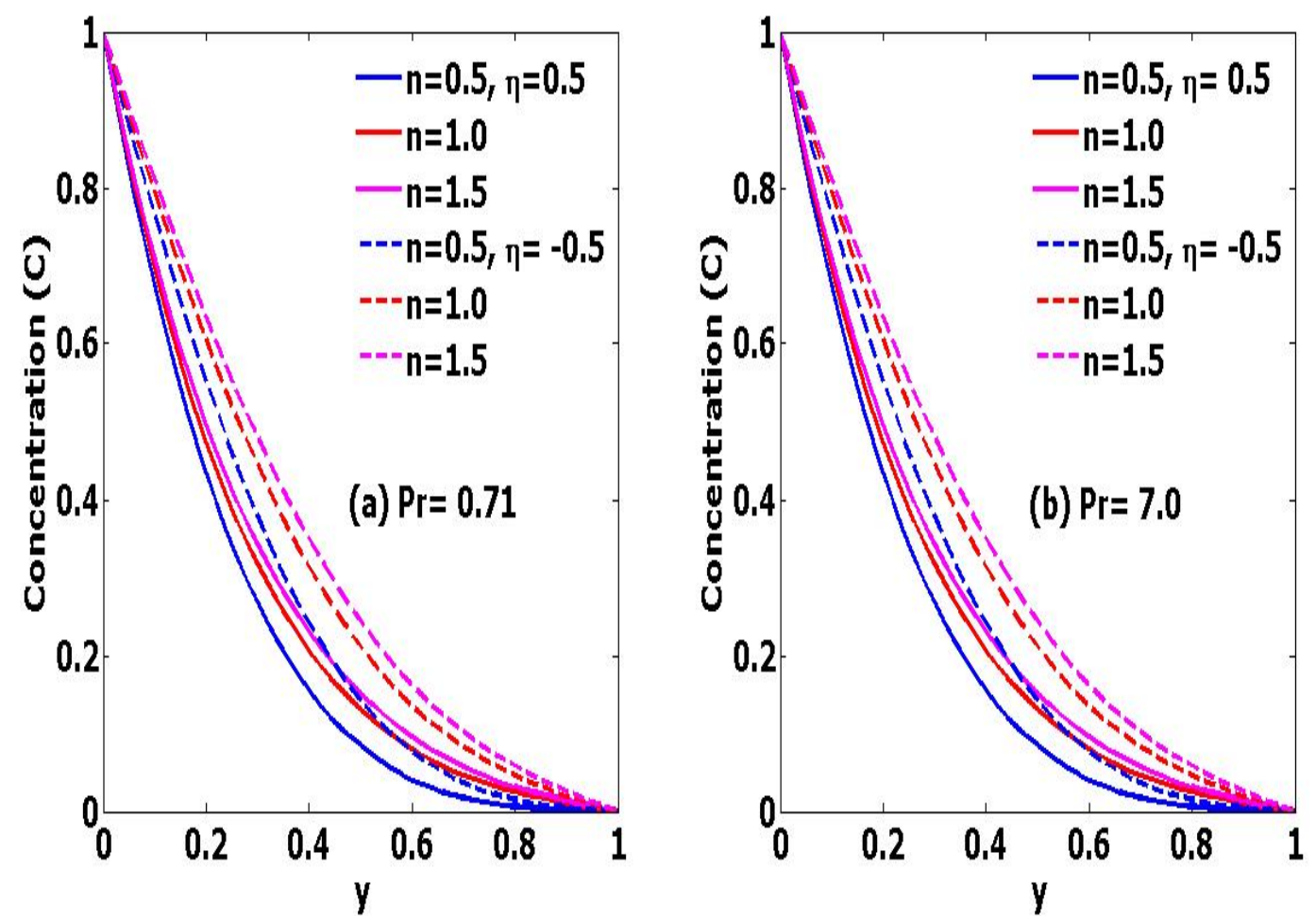

Figure 13: Effect of Order of chemical reaction parameter $(n)$ on Concentration profiles

The values of the skin-friction $\tau_{0}$ and $\tau_{1}$ of the transient part of the flow are entered in the Table 1 below for various values of the fluid parameter $D a, \operatorname{Re}, M, N$ and $\lambda$. The increase in Darcy number $(D a)$ increases the skin friction $\tau_{0}$ at the plate $y=0$ and $\tau_{1}$ at the plate $y=1$. Increase in Reynolds number also increases $\tau_{0}$ and $\tau_{1}$. Increase in the magnetic parameter $(M)$ both $\tau_{0}$ and $\tau_{1}$ decline. Increase of sustention parameter $(\mathrm{N})$ influence the skin-friction at the plate $y=0$ and $y=1$. Increase in variable viscosity parameter $(\lambda)$ increases $\tau_{0}$ but $\tau_{1}$ remains unchanged.

Table 1: Skin friction $C_{f}$ at the plate with $D a, M, N, S c$, for $\operatorname{Pr}=0.71$

\begin{tabular}{|c|c|c|c|c|c|c|}
\hline Da & $\operatorname{Re}$ & $\mathrm{M}$ & $\mathrm{N}$ & $\lambda$ & $\tau_{0}=\left.\frac{\partial u}{\partial y}\right|_{y=0}$ & $\tau_{1}=\left.\frac{\partial u}{\partial y}\right|_{y=1}$ \\
\hline 0.01 & 0.1 & 10 & 100 & 0.01 & 2.5794 & 0.0126 \\
\hline
\end{tabular}

\begin{tabular}{|l|l|l|l|l|l|l|}
\hline 0.02 & 0.1 & 10 & 100 & 0.01 & 3.6568 & 0.0229 \\
\hline 0.03 & 0.1 & 10 & 100 & 0.01 & 4.3956 & 0.0312 \\
\hline 0.01 & 0.1 & 10 & 100 & 0.01 & 2.5794 & 0.0126 \\
\hline 0.01 & 0.2 & 10 & 100 & 0.01 & 3.6568 & 0.0229 \\
\hline 0.01 & 0.3 & 10 & 100 & 0.01 & 4.3956 & 0.0312 \\
\hline 0.01 & 0.1 & 10 & 100 & 0.01 & 2.5794 & 0.0126 \\
\hline 0.01 & 0.1 & 20 & 100 & 0.01 & 2.5657 & 0.0124 \\
\hline 0.01 & 0.1 & 30 & 100 & 0.01 & 2.5522 & 0.0123 \\
\hline 0.01 & 0.1 & 10 & 100 & 0.01 & 2.5794 & 0.0126 \\
\hline 0.01 & 0.1 & 10 & 200 & 0.01 & 5.1338 & 0.0251 \\
\hline 0.01 & 0.1 & 10 & 300 & 0.01 & 7.6882 & 0.0376 \\
\hline 0.01 & 0.1 & 10 & 100 & 0.01 & 2.5794 & 0.0125 \\
\hline 0.01 & 0.1 & 10 & 100 & 0.02 & 2.5903 & 0.0125 \\
\hline 0.01 & 0.1 & 10 & 100 & 0.03 & 2.6012 & 0.0125 \\
\hline
\end{tabular}

Table 2 shows the influence of $F, \phi, E c$ and $\gamma$ on the rate of heat transfer for unsteady parts of the flow. It is observed that the increase in the radiation parameter $(F)$ decreases 
$N u_{0}$ at the plate $y=0$ but increases $N u_{1}$ at the plate $y=1$. Increasing the heat source parameter $\phi$, the Nusselt number at $\mathrm{y}=0$ and $\mathrm{y}=1$ remain unchanged. Increase in the Eckert number $(E c)$ increases $N u_{0}$ but decreases $N u_{1}$. The increase in value of variable thermal conductivity parameter ( $\gamma$ ) decreases both $N u_{0}$ and $N u_{1}$.

Table 2: Nusselt number at the plate with $F, \phi, E c$, and $\gamma$ for

$$
\operatorname{Pr}=0.71
$$

\begin{tabular}{|c|c|c|c|c|c|}
\hline $\mathrm{F}$ & $\phi$ & $E c$ & $\gamma$ & $N u_{0}=\left.\frac{\partial T}{\partial y}\right|_{y=0}$ & $N u_{1}=\left.\frac{\partial T}{\partial y}\right|_{y=1}$ \\
\hline 1.0 & 0.01 & 0.01 & 0.01 & 3.6259 & 0.0513 \\
\hline 2.0 & 0.01 & 0.01 & 0.01 & 2.9068 & 0.1747 \\
\hline 3.0 & 0.01 & 0.01 & 0.01 & 2.4971 & 0.3042 \\
\hline 1.0 & 0.01 & 0.01 & 0.01 & 3.6259 & 0.0513 \\
\hline 1.0 & 0.02 & 0.01 & 0.01 & 3.6256 & 0.0513 \\
\hline 1.0 & 0.03 & 0.01 & 0.01 & 3.6252 & 0.0513 \\
\hline 1.0 & 0.01 & 0.01 & 0.01 & 3.6259 & 0.0513 \\
\hline 1.0 & 0.01 & 0.02 & 0.01 & 3.6257 & 0.0513 \\
\hline 1.0 & 0.01 & 0.03 & 0.01 & 3.6254 & 0.0513 \\
\hline 1.0 & 0.01 & 0.01 & 0.01 & 3.6259 & 0.0513 \\
\hline 1.0 & 0.01 & 0.01 & 0.02 & 4.1525 & 4.1525 \\
\hline 1.0 & 0.01 & 0.01 & 0.03 & 4.1423 & 4.1423 \\
\hline
\end{tabular}

Table 3 depicts the values of the concentration gradient for the unsteady part of the flow with respect to various values of $S c, K r$ and $n$. It is noticed that the rise in the values of Schmidt number Sc decreases very rapidly for both plates at the plate $y=0$ and at the plate $y=1$. It is also deduced that the increase in chemical reaction parameter $\mathrm{Kr}$ decreases $S h_{0}$ and $S h_{1}$. It is also inferred from Table 3 that increase in the order of chemical reaction parameter influences both $S h_{0}$ and $S h_{1}$.

Table:3 Sherwood number $S h$ at the plate with $S c, K r$ for

$$
\operatorname{Pr}=0.71
$$

\begin{tabular}{|c|c|c|c|c|}
\hline$S c$ & $K r$ & $n$ & $S h_{0}=\left.\frac{\partial C}{\partial y}\right|_{y=0}$ & $S h_{1}=\left.\frac{\partial C}{\partial y}\right|_{y=1}$ \\
\hline 0.22 & 10.0 & 1.0 & 4.1629 & 4.1629 \\
\hline 0.78 & 10.0 & 1.0 & 1.9078 & 1.9078 \\
\hline 0.94 & 10.0 & 1.0 & 1.4679 & 1.4679 \\
\hline 0.22 & 10.0 & 1.0 & 4.1629 & 4.1629 \\
\hline
\end{tabular}

\begin{tabular}{|l|l|l|l|l|}
\hline 0.22 & 20.0 & 1.0 & 3.9159 & 3.9159 \\
\hline 0.22 & 30.0 & 1.0 & 3.6823 & 3.6823 \\
\hline 0.22 & 10.0 & 1.0 & 4.1629 & 4.1629 \\
\hline 0.22 & 10.0 & 1.5 & 4.2143 & 4.2143 \\
\hline 0.22 & 10.0 & 2.0 & 4.2495 & 4.2495 \\
\hline
\end{tabular}

\section{CONCLUSION}

In this study we have examined on effects of some fluid properties on unsteady conductive free convective fluid with suction/injection. It is resolved that the growth in the Schmidt number $(S c)$, variable viscosity parameter $(\lambda)$ and the magnetic parameter $(M)$ contributes to reduction in the velocity field. It is further resolved that with increase in the buoyancy parameter and the radiation parameter, the velocity field increases. The Skin friction decreases with growing of magnetic parameter $(M)$ whereby Skin friction growths with the increasing value of buoyancy parameter $(N)$ and variable viscosity parameter $(\lambda)$.

\section{REFERENCES}

[1]. Bachelor GK, Shen C. Thermophoretic deposition of particles in gas flowing over cold surfaces. J Colloid Interface Science 1985; 107:21-37.

[2]. Anyakoha MW. New School Physics. 3rd Edition Africana First Publisher Plc; 2010. p. 36-51. http://dx.doi.org/10.1016/00219797 (85)90145-6.

[3]. Andersson HI, Hansen OR, Holmedal B. Diffusion of a chemically reactive species from a stretching sheet. Int J Heat Mass Transfer 1994;

[4]. G. Venkata Ramana Reddy Ali J Chamkha , (2015),"Lie group analysis of chemical reaction effects on MHD free convection dissipative fluid flow past an inclined porous surface", International Journal of Numerical Methods for Heat \& Fluid Flow, 25(7); 1557 - 1573.

[5]. Animasaun I.L.( 2015), "Effects of thermophoresis, variable viscosity and thermal conductivity on free convective heat and mass transfer of non-Darcian MHD dissipative Casson fluid flow with suction and $\mathrm{n}^{\text {th }}$ order of chemical reaction;"Journal of the Nigerian Mathematical Society"34 11-31.

[6]. Krishna M.V., Sawarnalathamma B.V. , Chamkha A.J. (2019), "Investigations of Soret, Joule and Hall effects on MHD rotating mixed convective flow past an infinite vertical porous plate;"Journal of Ocean Engineering and Science”, 4, 263-275.

[7]. Gnaneswara M. R. and Bhaska N. R (2009). "Unsteady MHD convective heat and mass Transfer past semi-infinite vertical porous plate with variable viscosity and thermal conductivity, "International Journal of Applied Mathematics and Computation" 1(2), 104-117.

[8]. Oyem O.A., Omowaye A.J. and Koriko O.K.( 2015), "MHD Free Convective Heat Transfer on Reacting Flow over a Vertical Plate with Constant Thermal Conductivity; "International Journal of Applied Science and Mathematical Theory"1(8), 61-70.

[9]. Kesavaiah D. Ch., Satyanarayana P.V., Venkata Ramana S (2012), "Radiation Absorption, Chemical Reaction and Magnetic Field Effects On The Free Convection And Mass Transfer Flow Through Porous Medium With Constant Suction And Constant Heat Flux, "International Journal of Scientific Engineering and Technology"; 1,(6), 274-284. 
[10]. Durojaye M.O., Jamiu K.A and Ogunfiditimi F.O,( 2020), "Effects of Some Flow Parameters on Unsteady MHD Fluid Flow Past a Moving Vertical Plate Embedded in Porous Medium in the Presence of Hall Current and Rotating System", Asian Research Journal of Mathematics 16(6): 15-29.

[11]. Manjunatha S. and Gireesha B.J., (2015), "Effects of variable viscosity and thermal conductivity on MHD flow and heat transfer of a dusty fluid" Ain Shams Engineering Journal 7: 505-515.

[12]. Ali L., Liu X. and Ali B. (2020), "Finite Element Analysis of Variable Viscosity Impact on MHD flow and Heat Transfer in Nano fluid Using the Cattaneo-Christov model, "Coatings, 10(395), 5-19.

[13]. Mohammad Ali and Mohammad Shah Alam, (2014)" Soret and Hall effect on MHD flow, heat and mass transfer over a vertical stretching sheet in a porous medium due to heat generation", ARPN Journal of Engineering and Applied Sciences; 9(3);361371.

[14]. G.K. Bachelor, An Introduction to Fluid Mechanics, Cambridge University Press, London, 1987.

[15]. T.G. Meyers, J.P.F. Champin, M.S. Tshela, The flow of variable viscosity fluid between parallel plates with shear heating; Appl. Math. Model. 30 (2006) 799-815.

[16]. Gbadeyan J.A., Titiloye; E.O; Adeosun A.T;" Effect of variable thermal conductivity and viscosity on Casson Nanofluidflow with convective heating and velocity slip", Heliyon 6 (2020) e03076, www.elsevier.com/locate/heliyon.

[17]. Baruah I. and Hazarika G.C, (2017),"Effects of variable viscosity and thermal conductivity on unsteady micro polar fluid about a permeable cylinder under moving boundaries; International Journal of Engineering and Science; 6(9), 33-41.

[18]. Kiran Kumar, G., Srinivas, G., Babu, S., \& Srikanth, G. (2018) Effects of variable viscosity and thermal conductivity on MHD convective heat transfer of immiscible fluids in a vertical channel. International Journal of Engineering Sciences \& Research Technology;7(5), 73-79.

[19]. Chandra Babu S.R.R., Venkateswarlu S. and Lakshmi K.J., (2018) "Effect of Variable Viscosity and Thermal Conductivity on Heat and Mass Transfer Flow of Nanofluid over a Vertical Cone with
Chemical Reaction", International Journal of Applied Engineering Research; 13(18), 13989-14002.

[20]. Swain K., Parida S.K. and Dash G.C., (2018) "MHD Heat and Mass Transfer on Stretching Sheet with Variable Fluid Properties in Porous Medium", AMSE JOURNALS-AMSE IIETA publication2017-Series: Modelling B; 86(3); 706-726.

[21]. Patil P.M. and Kulkarmi P.S. (2008), "Effects of chemical reaction on free convective flow of a polar fluid through a porous medium in the presence of internal heat generation;International Journal of Thermal Sciences 47 1043-1054.

[22]. Cussler EL. Diffusion Mass Transfer in Fluid Systems. London: Cambridge University Press; 1988.

[23]. Uwanta I.J., Halima U (2014) "Convective Heat and Mass Transfer Flow Over A Vertical Plate with Nth Order Chemical Reaction in A Porous Medium; International Journal of Scientific Engineering and Technology; 3(2), 172 - 185.

[24]. Raghunath K, Krishna M.V; RajuG.S.S (2016)," Heat and Mass Transfer on unsteady MHD flow of a Viscos-elastic fluid past an Infinite Vertical Oscillatory Porous plate" British Journal of Mathematics and Computer Science; 17(6); 1-18.

[25]. Mjankwi M.A., Masanja V.G., Mureithi E.W. James M.N., "Unsteady MHD Flow of Nano fluid with Variable Properties over a Stretching Sheet in the Presence of Thermal Radiation and Chemical Reaction", International Journal of Mathematics and Mathematical Sciences Volume 2019, Article ID 7392459,14 pages.

[26]. Uwanta I.J. and Murtala M. (2014), "Heat Mass Transfer Flow past an Infinite Vertical Plate with Variable Thermal Conductivity, Heat Source and Chemical Reaction", International Journal of Engineering and Science, 3(5); 77-89.

[27]. Uwanta I.J. and Sani M. (2014), "Heat Mass Transfer Flow past an Infinite Vertical Plate with Variable Thermal Conductivity, Heat Source and Chemical Reaction", International Journal of Engineering and Science, 3(5); 77-89.

[28]. Jha BK, Isah BY, Uwanta IJ. Combined effect of suction/injection on MHD free convection flow in a vertical channel with thermal radiation. Ain Shams Eng J. 2018; 9(4):1069-1088.

[29]. Brewster, M.Q. (1992). Thermal Radiative Transfer and properties. John Wiley \& Sons. Inc., NewYork. 